Herz $2017 \cdot 42: 564$

DOI 10.1007/s00059-017-4607-9

Published online: 11 August 2017

๑) Springer Medizin Verlag GmbH 2017

CrossMark

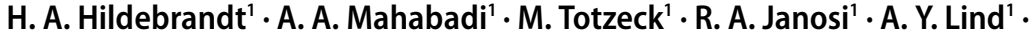
T. Schlosser ${ }^{2} \cdot$ M. Forsting ${ }^{2} \cdot$ T. Rassaf' $^{\prime}$ P. Kahlert ${ }^{1}$

'West-German Heart and Vascular Center Essen, Department of Cardiology and VascularMedicine, Essen University Hospital, Essen, Germany

${ }^{2}$ Institute for Diagnostic and Interventional Radiology and Neuroradiology, Essen University Hospital, Essen, Germany

\section{Erratum to: Imaging for planning of transcatheter aortic valve implantation}

\section{Erratum to:}

Herz (2017)

DOI 10.1007/s00059-017-4587-9

In the above mentioned article, two authors were missing. The correct list of authors is as follows:

H.A. Hildebrandt ${ }^{1}$, A.A. Mahabadi ${ }^{1}$, M. Totzeck ${ }^{1}$, R.A. Janosi ${ }^{1}$, A.Y. Lind ${ }^{1}$, T. Schlosser ${ }^{2}$, M. Forsting ${ }^{2}$, T. Rassaf ${ }^{1}$, P. Kahlert ${ }^{1}$

${ }^{1}$ West-German Heart and Vascular Center Essen, Department of Cardiology and Vascular Medicine, Essen University Hospital, Essen, Germany

${ }^{2}$ Institute for Diagnostic and Interventional Radiology and Neuroradiology, Essen University Hospital, Essen, Germany

The authors apologize for this mistake.

\section{Corresponding address}

Univ.-Prof. Dr. med. P. Kahlert

West-German Heart and Vascular Center Essen, Department of Cardiology and VascularMedicine, Essen University Hospital Hufelandstr. 55, 45122 Essen, Germany philipp.kahlert@uk-essen.de 